\title{
Winkelstabile Implantate - Einsatzmöglichkeiten am distalen Radius
}

\author{
Stefan Matschke, Andreas Wentzensen
}

\section{Zusammenfassung}

Das LCP-Konzept mit winkelstabil verankerten Plattenschrauben hat für die Versorgung der distalen Radiusfraktur einen wesentlichen Fortschritt gebracht. Stellten bislang für den komplikationsarmen radio-palmaren Zugang ausschließlich Flexionsfrakturen eine Indikation dar, so kann unter Verwendung der LCP das Indikationsspektrum auf extraartikuläre als auch artikuläre Extensionsfrakturen (90\% aller Frakturen) ausgeweitet werden. Somit lassen sich alle distalen Radiusfrakturen von der A3- bis zur C3- Fraktur, die mit einer Plattenosteosynthese versorgt werden können, mit einer LCP versorgen [6]. Die bislang vorliegenden Ergebnisse einer prospektiv angelegten Multicenterstudie mit 9 teilnehmenden Einrichtungen bei der Behandlung distaler Radiusfrakturen (197 distale Radiusfrakturen, hiervon waren 165 mit einer LCP 3,5 mm versorgt) zeigten lediglich in 5 Fällen nach 6 Monaten einen sekundären Korrekturverlust. Insbesondere fand sich ein sicherer Schrauben- und Plattenhalt im osteoporotischen Knochen, so dass auch gerade bei diesen Problemfrakturen ein komplikationsloser Verlauf beobachtet wurde.

\section{Einleitung}

\section{Prinzip}

Das Prinzip der LCP beruht auf der winkelstabilen Verriegelung der Schraubenköpfe in dem Gewinde der Platte, weshalb ein direkter Periostkontakt der Platte nicht erforderlich ist (Lastübertragung erfolgt nicht über Haftreibung wie bei der herkömmlichen Plattenosteosynthese), Abb. 1.

Durch die Verbindung zwischen Kopfverriegelungsschraube und der ersten Kortikalis entsteht ein stabiler Rahmen der den Einsatz auch monokortikaler Schrauben ermöglicht, die einer axial einwirkenden Kraft widerstehen.

Die LCP-Platte funktioniert nicht als Abstützplatte, sondern als „Winkelplatte“.

Probleme nicht „winkelstabiler“ Implantate stellen Repositionsverluste durch

OP-JOURNAL 2004; 20: 18-26

(c) Georg Thieme Verlag KG Stuttgart · New York rückläufige Schrauben, Abkippungen distaler Fragmente oder Sinterungen der Fraktur dar, insbesondere beim osteoporotischen Knochen [7].

Ziel der neuen Implantate ist es einerseits Korrekturverluste zu verhindern, insbesondere auch bei Frakturen mit osteoporotischen Knochen. Des Weiteren ermöglicht die LCP, dass auch Extensionsfrakturen über einen komplikationslosen radio-palmaren Zugang versorgt werden können. Probleme des dorsalen Zugangs wie Strecksehnenrupturen [4], eine Tendinitis durch ständige Reizungen des Implantats bzw. eine obligate Metallentfernung, entfallen.
Anwendungsbeispiele für die Osteosynthese von palmar mit winkelstabiler Platte

Mit den bislang zur Verfügung stehenden konventionellen 3,5-mm-Formplatten bestand die Indikation zur palmaren Plattenosteosynthese für alle nach palmar dislozierten Frakturen (Typ Goyrand Smith) sowie bei palmar dislozierten Kantenfragmenten (Typ B3, reversed Barton) $[1,2]$.

Die gegenwärtig verfügbaren winkelstabilen Platten (3,5 mm oder $2,4 \mathrm{~mm}$ ) ermöglichen hingegen auch nach dorsal dislozierte extraartikuläre bzw. artikuläre Frakturen von palmar zu versorgen.

Diese Osteosynthesen verbinden den Vorteil des einfacheren Zugangs von palmar (Henry), der günstigen Plattenlage (keine Strecksehnenirritationen) [3] mit dem Vorteil der winkelstabilen Schraubenverankerung. Hierdurch entfällt die häufige Notwendigkeit von Spongiosatransplantationen, die bei konventionellen Plattenosteosynthesen bei Frakturen mit metaphysären Defekten erforderlich ist.

Da die LCP-Formplatte nicht als Abstützplatte wie bei der dorsalen Plattenosteosynthese funktioniert, sondern als „Winkelplatte“ ergeben sich auch zwei verschiedene Möglichkeiten der Reposition.

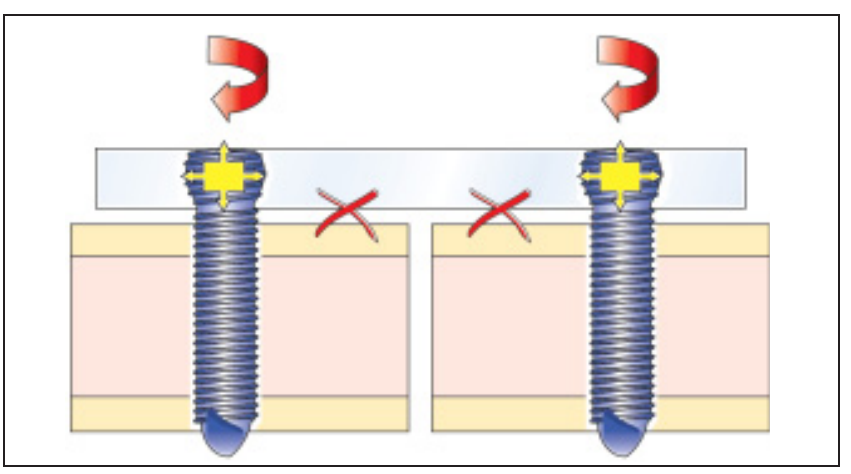

Abb. 1 Schematische Darstellung der Plattenlage zum Periost unter Verwendung von KVS. 

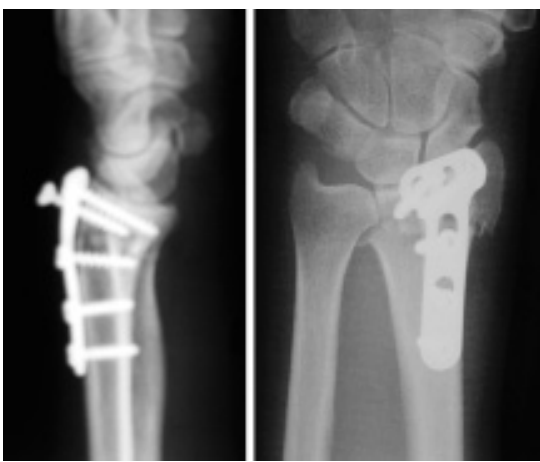

Abb. 2 Lockerung des Implantats durch rückläufige Schraube bei nicht winkelstabiler Plattenosteosynthese.

1. Reposition über den T-Schenkel der LCP Formplatte

Um eine physiologische Palmarinklination $\mathrm{zu}$ erreichen, muss der T-Schenkel der LCP-Formplatte zunächst mit der Zange vorgebogen werden (Abb. 3c).

Handelt es sich um ein intaktes metaphysäres Fragment (A3-Fraktur, Abb. 3a,b), so wird nach dem Vorbiegen des T-Schenkels der Formplatte zunächst dieser TSchenkel mit winkelstabilen Schrauben in der Metaphyse fixiert (Abb. 3d-e). Unter Extension und Flexion der Hand wird unter Ausnutzung des langen Schenkel der Formplatte die Fraktur reponiert und die Platte an den Radiusschaft mit Schrauben befestigt. Hierbei stellt sich automatisch die physiologische Palmarinklination ein (in Abhängigkeit von dem Vorbiegen des T-Schenkels der Formplatte) (Abb.3f-g).

\section{Reposition über den langen Schenkel} der LCP-Formplatte

Bei Frakturen, bei denen die Metaphyse in sich frakturiert ist (C-Frakturen, Abb. 4a,b), wird zunächst der lange Schenkel der LCP-Formplatte in einem Gleitloch am Radiusschaft fixiert. Die korrekte Lage der Platte wird unter BV-Kontrolle überprüft (Abb.4c,d).

Die Bohrbüchse wird in das Plattengewinde am T-Schenkel eingeschraubt.

Unter Extension und Flexion der Hand Reposition der Fraktur an den T-Schenkel der LCP-Formplatte. Unter Röntgenkontrolle erfolgt das Vorbohren für die selbstschneidenden Schrauben, die subchondral ihren besten Halt finden (Abb.4c). Gegebenenfalls muss die Plattenlage nochmals durch Lockern der Schraube

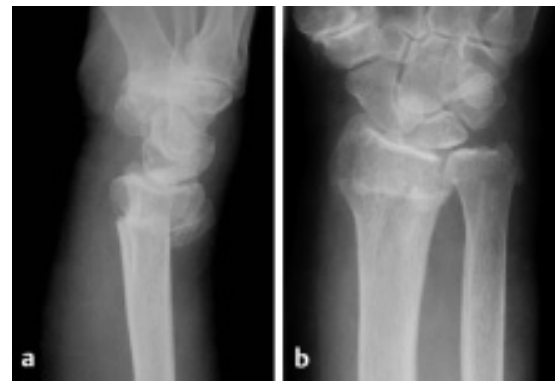

Abb.3a,b A3-Fraktur einer 78-jährigen Patientin nach häuslichem Sturz.

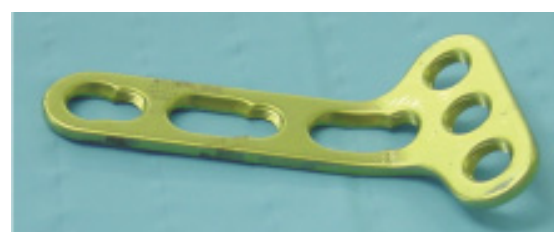

Abb.3c Der T-Schenkel der LCP-Formplatte wurde mit einer Zange vorgebogen.

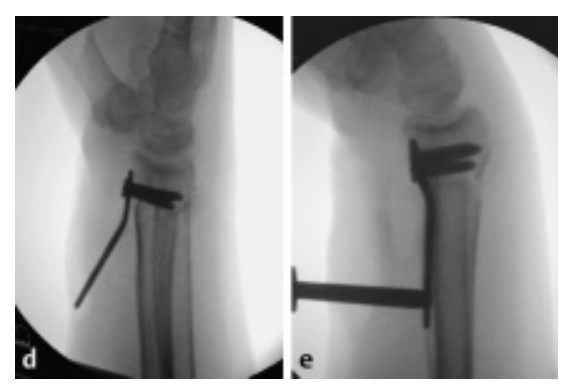

Abb.3d,e Einbringen der winkelstabilen Schrauben in den T-Schenkel der LCP-Formplatte. (e) Aufrichten der Fraktur unter Extension und Flexion der Hand und das Andrücken des langen Schenkels der LCP Formplatte an den Schaft.
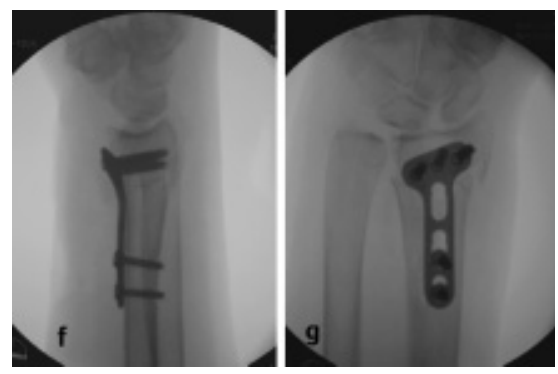

Abb.3f,g Postoperative Versorgungsbilder der A3-Fraktur bei physiologischer Palmarinklination von $12^{\circ}$ und palmarer Plattenlage.
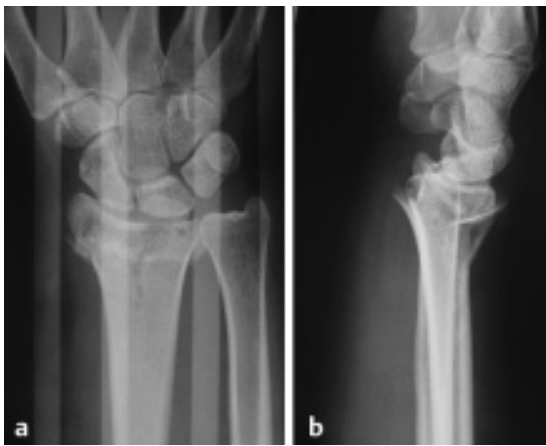

Abb.4a,b C2-Fraktur einer 46-jährigen Frau.

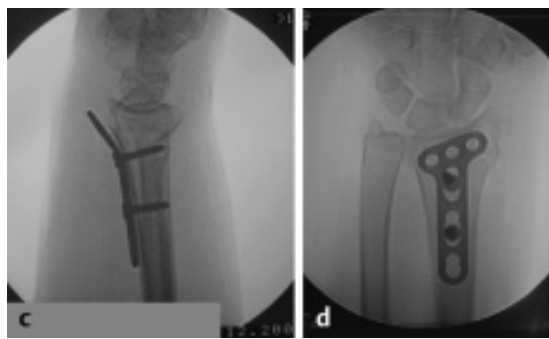

Abb.4c, d Zunächst Fixieren des langen Schenkels der LCP-Formplatte am Radiusschaft im Gleitloch.

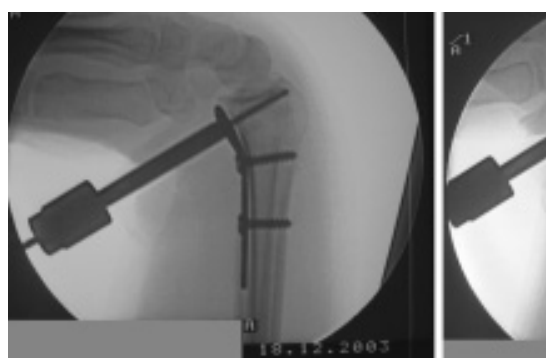

Abb.4e,f Unter Extension und Flexion der Hand Reposition der Fraktur an den T-Schenkel der LCP-Formplatte, Bohren für die selbstschneidenden Schrauben über die aufgesetzte Bohrbüchse. Nach Einbringen der ersten selbstschneidenden Schraube gleiches Vorgehen für die anderen winkelstabilen Schrauben.

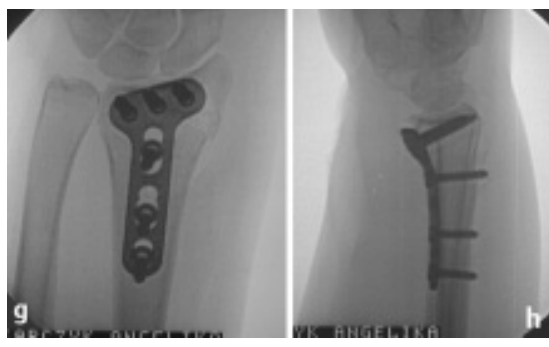

Abb.4g,h Postoperatives Versorgungsbild der C2-Fraktur. 


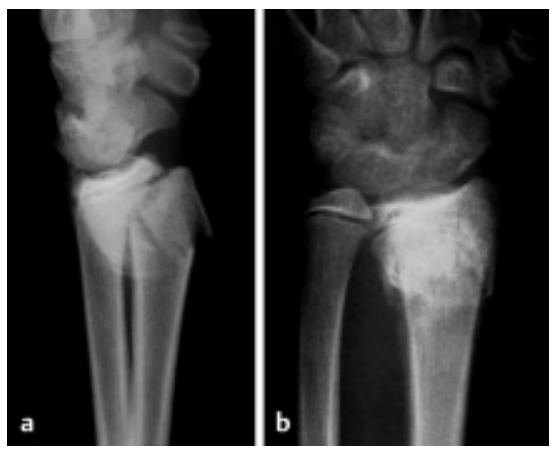

Abb.5a, b Mit dorsaler Fehlstellung von $20^{\circ}$ verheilte distale Radiusfraktur (Aitgen I) bei einem 14-jährigen Jungen nach konservativer Therapie.

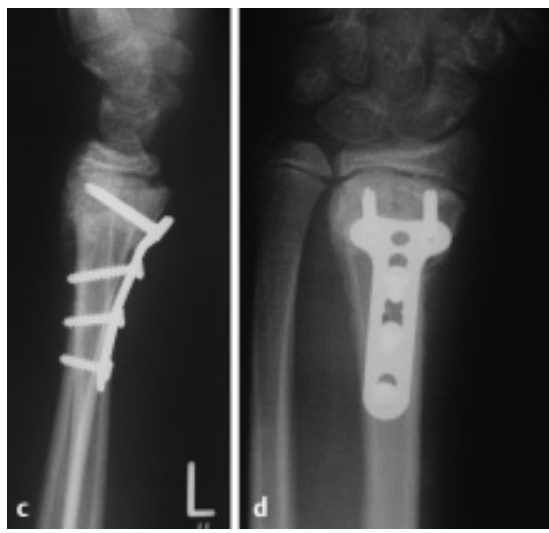

Abb.5c, d Postoperative Versorgungsbilder mit einer 3,5-mm-LCP. Die Korrektur erfolgte über einen beugeseitigen Zugang mit Osteotomie proximal der Epiphysenfuge. Nach Vorbiegen der Formplatte Einbringen der winkelstabilen Schrauben in den T-Schenkel der Formplatte und Reposition der Fehlstellung durch das Andrücken des langen Schenkels der 3,5-mm-LCP an den Schaft.

im Gleitloch korrigiert werden. Nach Einbringung der winkelstabilen Schrauben wird die Repositionsstellung gehalten (Abb.4d,e).

Weitere Anwendungsmöglichkeiten für den Einsatz der winkelstabilen LCP stellen Frakturen dar, die in einer Fehlstellung verheilt sind und eine Korrekturosteosynthese erfordern. Hierbei muss je nach vorliegender Situation entschieden werden, ob eine alleinige Osteotomie und Korrektur ausreichend ist wie im Beispiel Abb. 5 oder eine Aufrichtung durch das zusätzliche Einfügen eines autologen Beckenkammblocks erreicht wird.
Patientenkollektiv und Beobachtungszeitraum

Im Rahmen einer prospektiven Multicenterstudie über Behandlungsergebnisse distaler Radiusfrakturen mit einer LCP wurden von 9 teilnehmenden Einrichtungen bis zum 5.9.2003 195 Patienten erfasst, 125 Frauen und 70 Männer. Die Anzahl der Frakturen beträgt 197.

Entsprechend der AO-Klassifikation handelt es sich um 73 A-Frakturen, 21 B-Frakturen und 103 C-Frakturen.

Das Durchschnittsalter bei den Männern lag bei 45,3 Jahren (21 - 78 Jahre), das der Frauen bei 60,7 Jahren (20-81 Jahre).

Die Operation erfolgte durchschnittlich nach 3,6 Tagen (1-13 Tage).

Die Nachuntersuchungen erfolgen nach 6 Wochen, 6 Monaten, 1 bzw. 2 Jahren.

In 91 Fällen war die Frakturhand rechts lokalisiert, links in 104 Fällen (2 Angaben fehlend).

In 155 Fällen erfolgte der Zugang palmarseitig, dorsal wurde in 42 Fällen zugegangen.

Die Operationen wurden in durchschnittlich 73 min durchgeführt (Min 22-Max 220).

Hierbei wurde mit dem Bildwandler 115 Sekunden durchleuchtet (Min 0,4-Max 721).

\section{Unfallart}
38 hoch- und
- 157 niederenergetische Traumata;
- 61 daheim,
- 66 beim Sport oder in der Freizeit,
- 26 im Straßenverkehr,
- 22 Arbeitsunfälle und
- 20 nicht näher bezeichnete Stürze

In $1 / 4$ der Fälle handelte es sich um hochenergetische Traumen, in $3 / 4$ der Fälle um niederenergetische Traumata.

Abb. 6 widerspiegelt den zunehmenden Anteil von Frauen zwischen 60 und 80 Jahren, wodurch sich das Überwiegen der niederenergetischen Verletzungen erklärt. Bei den Männern liegt eine in etwa gleiche Häufung der Frakturen in den entsprechenden Altersklassen bis zum 65. Lebensjahr vor
Bei den Verletzungen überwiegen die CFrakturen mit 103 Fällen, wobei die C2Fraktur in 60 Fällen ebenso häufig wie die A3-Fraktur auftrat. B-Frakturen waren erwartungsgemäß mit 21 Fällen am seltensten.

Sämtliche Operationen wurden von insgesamt 60 verschiedenen Operateuren durchgeführt. Ein Zusammenhang zwischen dem Ergebnis der Operation und dem Ausbildungsstand des Operateurs konnte nicht nachgewiesen werden.

- 53 Chirurgen führten 1 bis 5 Operationen durch

a 5 zwischen 6 bis 10 und

a je einer 12 bzw. 29 .

- 19 Eingriffe wurden vom Chefarzt durchgeführt

- 85 vom Oberarzt,

- 72 von einen Assistenten/Facharzt und

- 19 von einem Jungassistenten/AIP.

\section{Implantate}

165 Frakturen wurden mit einer LCP DR $3.5 \mathrm{~mm}$ behandelt (119 schräge T-Platten, 46 rechtwinklige T-Platten). In 21 Fällen kam die winkelstabile 2,7-mm-Platte zur Anwendung, als dorsale Phi-Platte hierbei bei 7 Frakturen. 11 Frakturen wurden über zwei kleine Zugänge radial und dorsal mit einer Doppelplatte versorgt.

Tab.1 gibt die Verteilung zwischen den verwendeten Kopfverriegelungsschrauben (KVS) und den konventionellen Schrauben im distalen Plattenanteil bei den mit der LCP 3,5 mm versorgten Frakturen wieder

Es wird deutlich, dass im distalen Plattenteil hauptsächlich allein KVS verwendet wurden und hierbei meistens 3 (110 Fälle) bzw. 2 Stück (35 Fälle).

Die Verteilung der gemeinsam verwendeten Kopfverriegelungsschrauben (KVS) und konventionellen Schrauben im proximalen Plattenanteil bei den mit der LCP $3.5 \mathrm{~mm}$ behandelten Frakturen gibt Tab. 2 wieder.

Aus der zweiten Tabelle ist ersichtlich, dass im proximalen Plattenteil die Verteilung der Schrauben wesentlich homogener ist, wobei in 70 Fällen gänzlich auf eine KVS verzichtet wurde. Am häufigsten wurden 2 KVS mit 1 konventionellen Schraube kombiniert (36 Fälle).

Bei 24 Frakturen (12,2\%) wurde aufgrund der bestehenden metaphysären Defektsituation die Notwendigkeit zur 


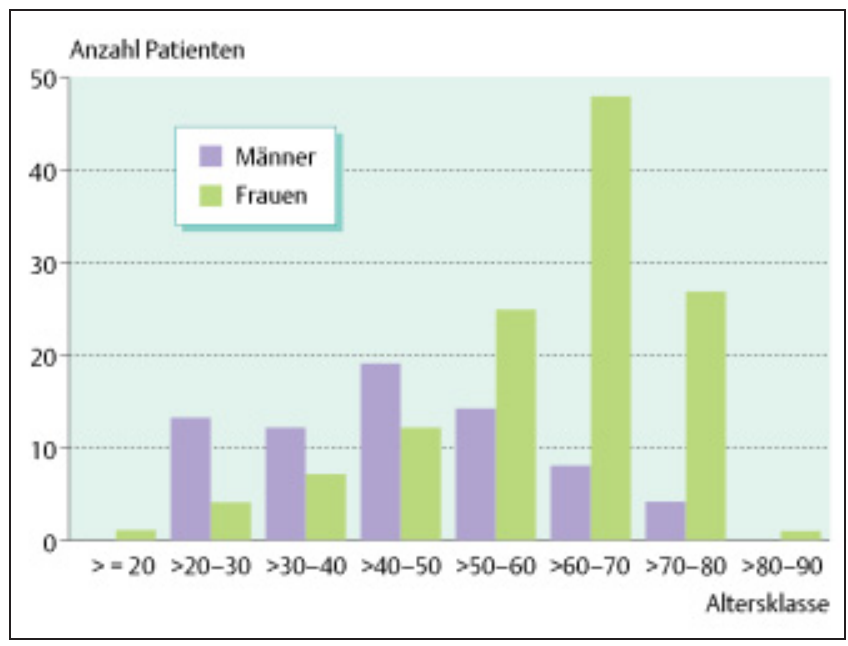

Abb. 6 Demografische Angaben Alters- und Geschlechtsverteilung.

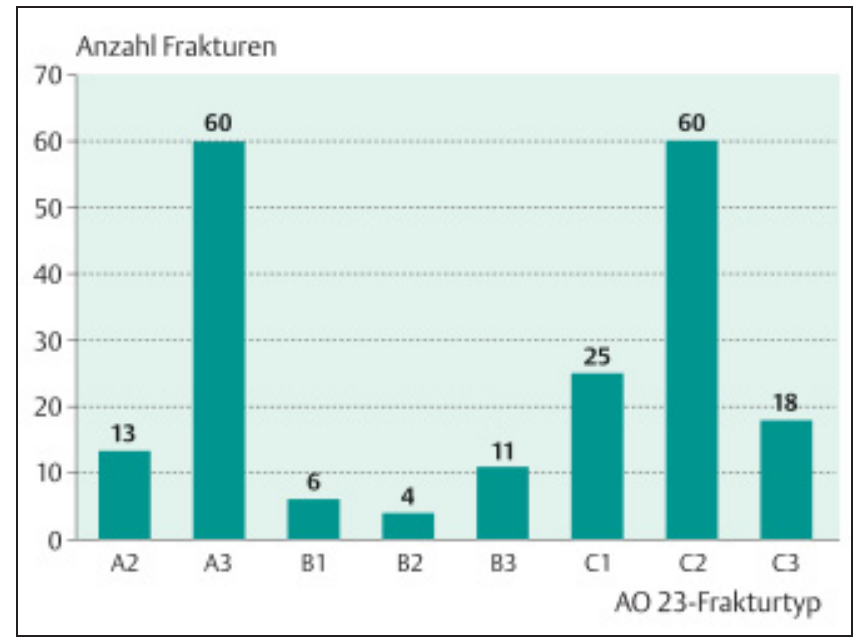

Abb. 7 Verteilung nach Frakturtyp.

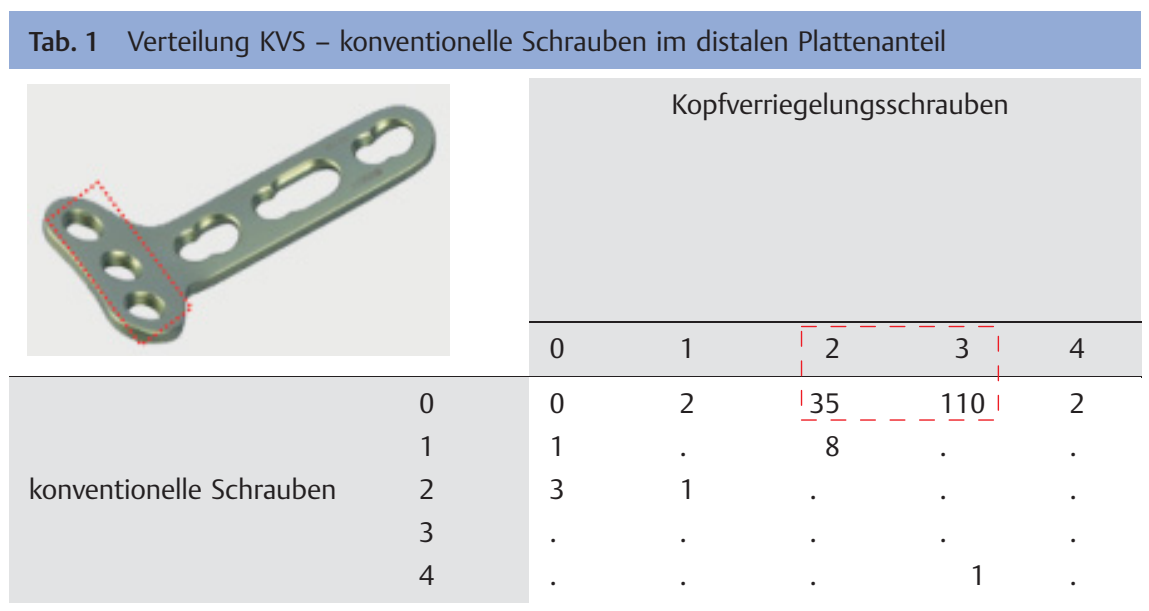

Tab. 2 Verteilung KVS - konventionelle Schrauben im proximalen Plattenanteil

\begin{tabular}{|c|c|c|c|c|c|c|}
\hline$\therefore$.... & & \multicolumn{5}{|c|}{ Kopfverriegelungsschrauben } \\
\hline & & 0 & 1 & 2 & 3 & 4 \\
\hline \multirow{5}{*}{ konventionelle Schrauben } & 0 & 0 & 1 & 12 & 11 & 1 \\
\hline & 1 & 1 & 12 & 36 & 4 & . \\
\hline & 2 & 30 & 14 & 1 & . & . \\
\hline & 3 & 38 & . & . & . & 1 \\
\hline & 4 & 1 & . & . & . & . \\
\hline
\end{tabular}

Spongiosatransplantation bzw. für einen Knochenersatz gestellt. Dies am häufigsten bei C2-Frakturen (11 Patienten). In 13 Fällen wurde autologe Spongiosa verwendet, in den übrigen 11 Fällen Homograft oder ein synthetischer Knochenersatz.
Gefragt wurde nach der Zufriedenheit des Chirurgen mit der Behandlung anhand der visuellen analogen Skala (VAS) (Abb.9).

Hierbei widerspiegelt der Wert 0 die größtmögliche Zufriedenheit und der Wert 10 die geringste Zufriedenheit. Bis auf 3 Chirurgen war die Zufriedenheit groß bzw. sehr groß. Bei den 3 Chirurgen mit Werten über 6 lassen sich anhand der Unterlagen folgende Gründe eruieren: eine tiefe Infektion nach Osteosynthese mit einer 2,7-mm-Platte, ein Patient hatte am Unfalltag eine Operation abgelehnt und konnte erst nach 6 Tagen operiert werden, beim dritten Patienten sind die Gründe nicht ersichtlich.

Die 6-Wochen-Verlaufskontrolle fand nach durchschnittlich 45 Tagen statt (Min 33, Max 81). Für das 6-Wochen-Follow-up wurden Angaben von 173 Patienten ausgewertet (Follow-up-Rate $88 \%$ ). Die Follow-up-Rate von $88 \%$ bezieht sich auf die Anzahl Patienten, deren Kontrolluntersuchung bis zum Tag der Auswertung (5.9.2003) hätte stattfinden sollen und deren Daten zur Auswertung vorlagen.

Gefragt wurden die Patienten nach den Schmerzen an der Bruchstelle. Die Schmerzen des Patienten wurden mit der visuellen analogen Schmerzskala (VAS) erfasst.

Dabei bedeutet der Wert 0 gar keine Schmerzen und der maximale Wert 10 absolut unerträgliche Schmerzen. Nur wenige Patienten hatten gar keine Schmerzen, die Mehrheit, rund zwei Drittel der Patienten, gab noch leichte Schmerzen an (Abb.10).

\section{Ergebnisse nach 6 Monaten}

122 Patienten wurden nach 6 Monaten, nach durchschnittlich 191 Tagen (Min 148, Max 299) nachuntersucht. Das entspricht einer Follow-up-Rate $63 \%$. 


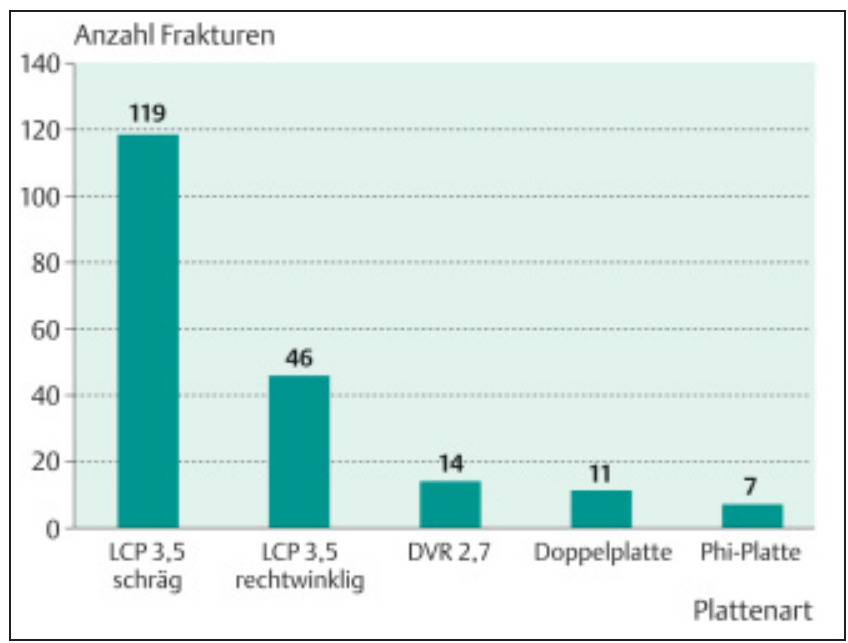

Abb. 8 verwendete Implantate.

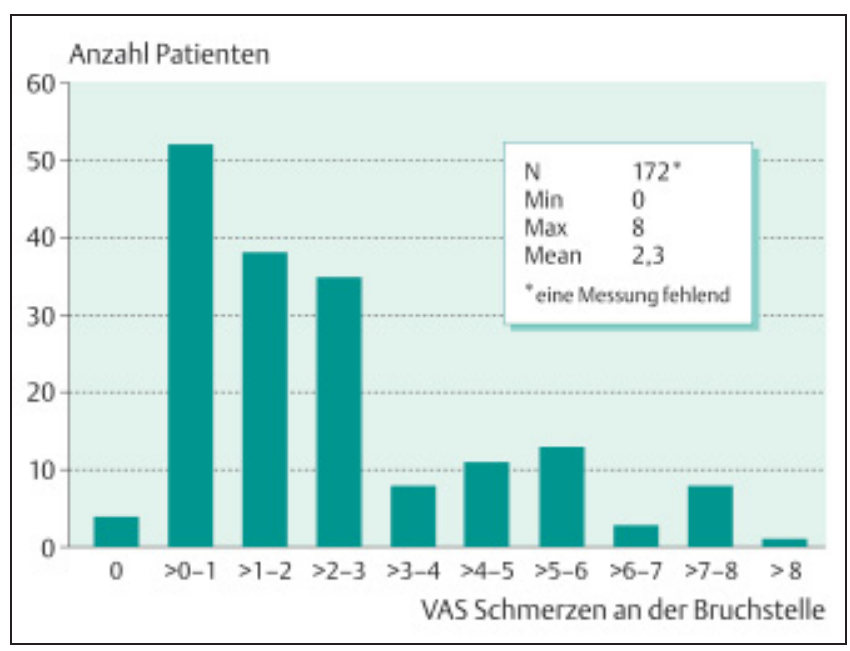

Abb.10 Schmerzen an der Bruchstelle.

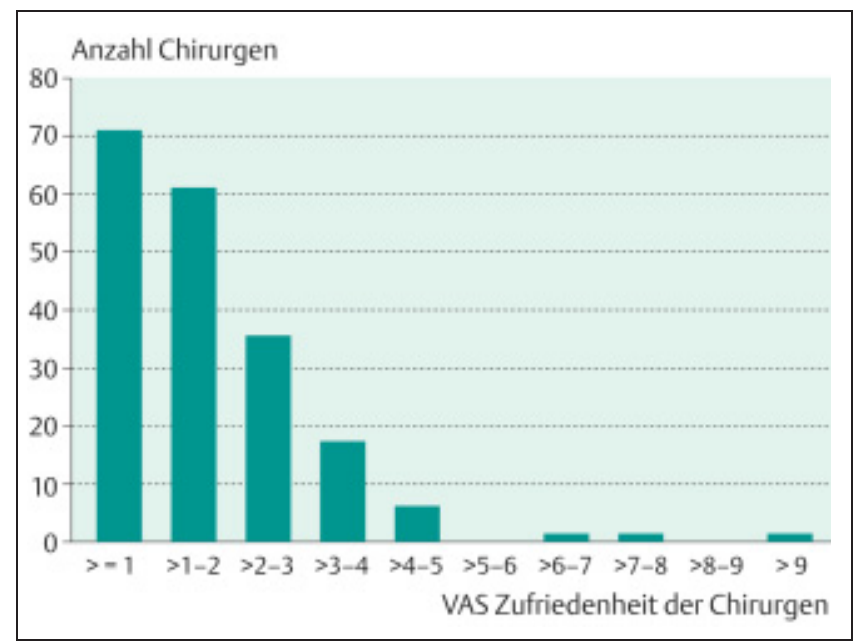

Abb.9 Zufriedenheit des Chirurgen mit der Behandlung.

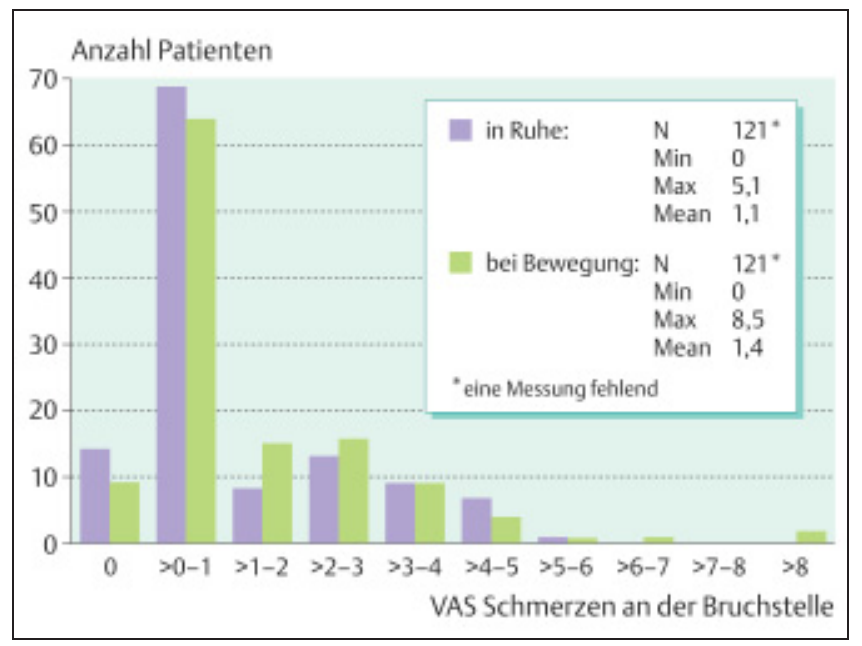

Abb.11 Schmerzen an der Bruchstelle nach 6 Monaten (VAS).
Die Follow-up-Rate von $63 \%$ bezieht sich auf die Anzahl Patienten, deren Kontrolluntersuchung bis zum Tag der Auswertung (5.9.2003) hätte stattfinden sollen und deren Daten zur Auswertung vorlagen.

Zu diesem Zeitpunkt war von Interesse zu erfahren, wie die Patienten die Schmerzen an der Bruchstelle in Ruhe und bei Bewegung anhand der visuellen Analogskala beurteilten.

Bei einem Durchschnittswert von 1.1 in Ruhe hatte die Mehrheit der Patienten nur noch geringfügige Schmerzen in Ruhe. Ebenso fand sich nur ein geringer Bewegungsschmerz bei einem Durchschnittswert von 1.4 (Abb.11).

Hinsichtlich der Bewegungsausmaße nach 6 Monaten fanden sich noch größere Bewegungseinschränkungen im Ver- gleich zur gesunden Seite bis maximal $15^{\circ}$ bei der Palmarflexion bzw. $12^{\circ}$ bei der Dorsalextension (Tab.3). Pro- bzw. Supination als auch Radial- und Ulnarduktion waren im Vergleich zur kontralateralen Seite nur noch geringfügig eingeschränkt.

Radiologisches Ergebnis nach 6 Monaten

Aufgeschlüsselt nach Frakturtypen zeigt sich nach 6 Monaten sowohl bei Typ A-, B- und C-Verletzungen ein normgerechter Radioulnarer Neigungswinkel (Tab.4). Lediglich bei den Typ-A-Fraktu-

\begin{tabular}{|c|c|c|c|c|c|c|}
\hline & \multicolumn{3}{|c|}{ ipsilateraler Arm } & \multicolumn{3}{|c|}{ kontralateraler Arm } \\
\hline & $\begin{array}{l}\text { Mini- } \\
\text { mum }\end{array}$ & $\begin{array}{l}\text { Maxi- } \\
\text { mum }\end{array}$ & Mean & $\begin{array}{l}\text { Mini- } \\
\text { mum }\end{array}$ & $\begin{array}{l}\text { Maxi- } \\
\text { mum }\end{array}$ & Mean \\
\hline Palmarflexion & 15 & 85 & 50 & 30 & 90 & 65 \\
\hline Dorsalextension & 10 & 90 & 55 & 15 & 85 & 67 \\
\hline Radialduktion & 5 & 45 & 19 & 10 & 50 & 25 \\
\hline Ulnarduktion & 5 & 60 & 28 & 10 & 65 & 34 \\
\hline Pronation & 30 & 90 & 81 & 90 & 90 & 84 \\
\hline Supination & 10 & 90 & 78 & 60 & 90 & 85 \\
\hline
\end{tabular}



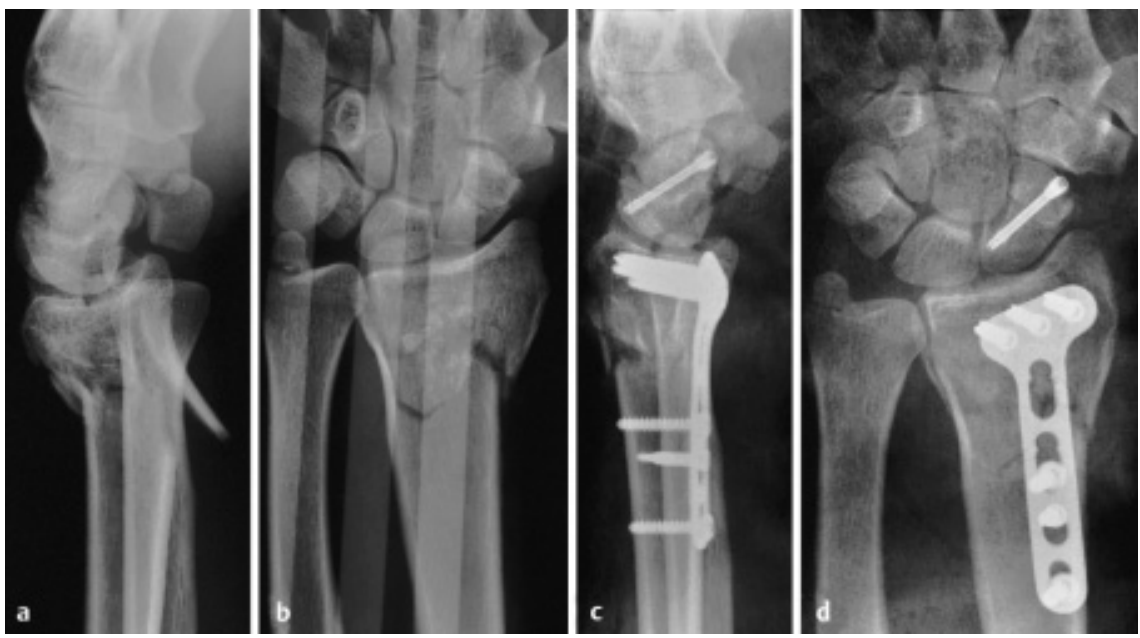

Abb.12a, b (a, b) C2-Fraktur mit begleitender Scaphoidfraktur präoperativ. (c, d) Versorgungsbild postoperativ mit 3,5-mm-LCP und Schraubenosteosynthese der begleitenden Scaphoidfraktur.
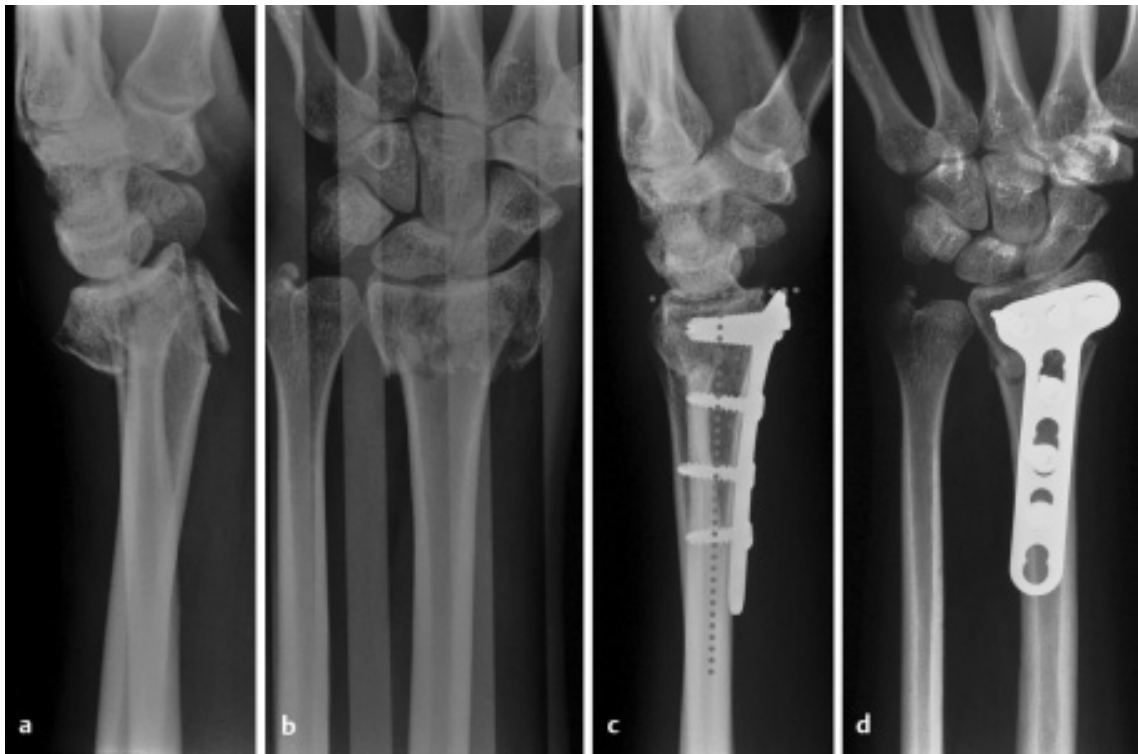

Abb.13 (a, b) C2-Fraktur präoperativ. (c, d) Versorgung mit palmarer 3,5-mm-LCP bei verbliebener Dorsalkippung.

Tab. 4 Radiologisches Ergebnis nach 6 Monaten

\begin{tabular}{|c|c|c|c|c|}
\hline & Norm & A & B & C \\
\hline $\begin{array}{l}\text { radioulnarer } \\
\text { Neigungswinkel }\left({ }^{\circ}\right)\end{array}$ & $22-23$ & 22.5 & 25,2 & 25.4 \\
\hline $\begin{array}{l}\text { palmarer } \\
\text { Neigungswinkel }\left({ }^{\circ}\right)\end{array}$ & $11-12$ & 6,5 & 11,7 & 13.1 \\
\hline Ulnavorschub & - & 2 & 0 & 3 \\
\hline
\end{tabular}

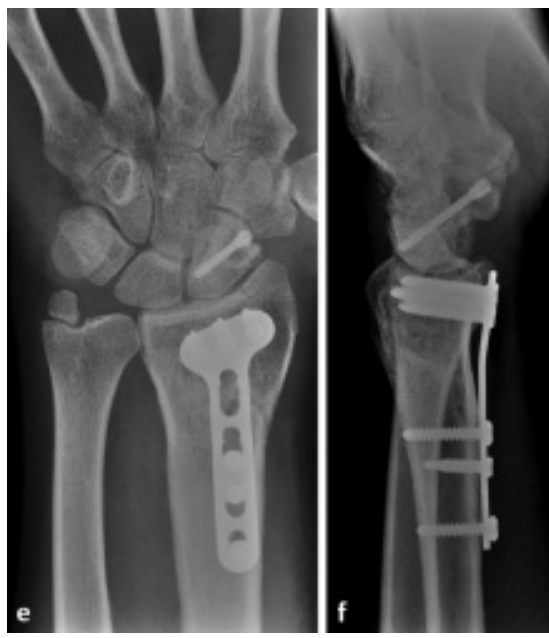

Abb.12e,f Verlauf nach 6 Monaten, knöcherne Konsolidierung ohne Korrekturverlust.

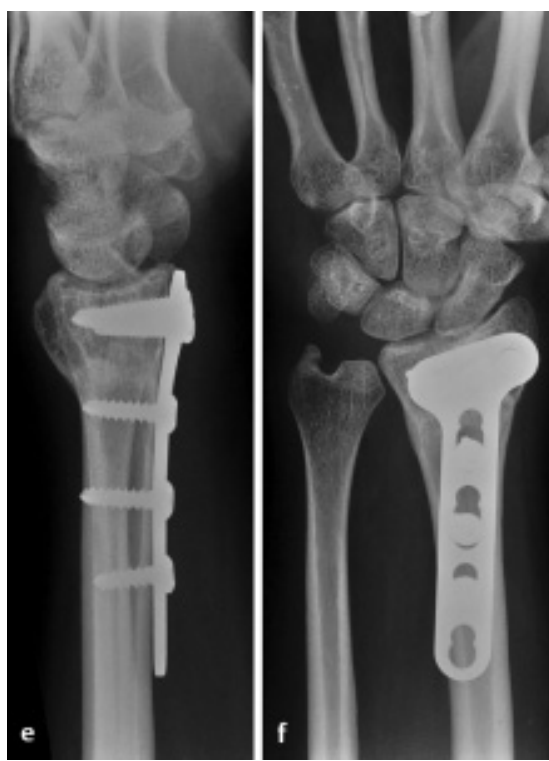

Abb.13e,f Radiologisches Ausheilungsbild nach 6 Monaten, kein weiterer Korrekturverlust.

ren war der palmare Neigungswinkel mit $6,5^{\circ}$ etwas verringert. Gründe hierfür lagen jedoch zumeist in einer intraoperativ nicht korrekt eingestellten Palmarinklination (Abb.13).

In 5 Fällen kam es nach 6 Monaten zu einem Korrekturverlust mit einem relativen Ulnavorschub bei 2 A3-Verletzungen bzw. bei 3 C-Verletzungen (Abb.14).

Zum 6-Monats-Follow-up erfolgte eine Evaluierung nach dem Gartland- und Werley-Score der 30\% subjektive und $70 \%$ objektive Parameter erfasst. Da einzelne Angaben fehlten, wurde der Score bei 118 Patienten erhoben. Hierbei fand sich folgendes Ergebnis: 


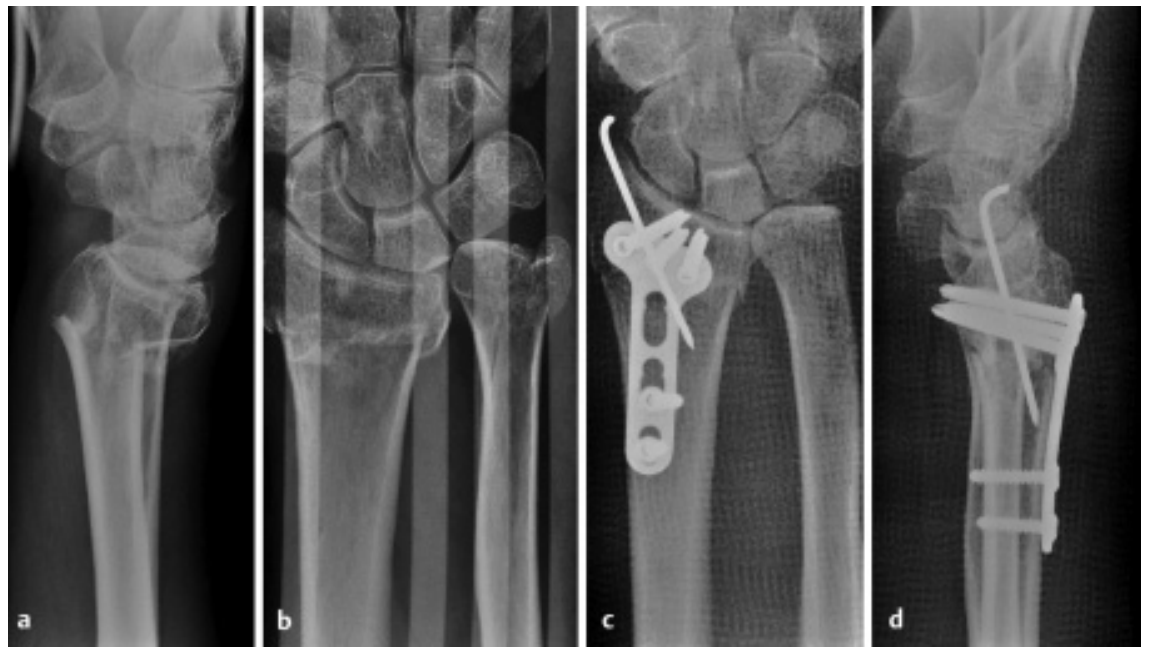

Abb.14 (a, b) A3-Fraktur präoperativ. (c, d) Versorgungsbilder postoperativ mit dorsaler 3,5$\mathrm{mm}-\mathrm{LCP}+\mathrm{K}$-Draht-Stabilisierung.

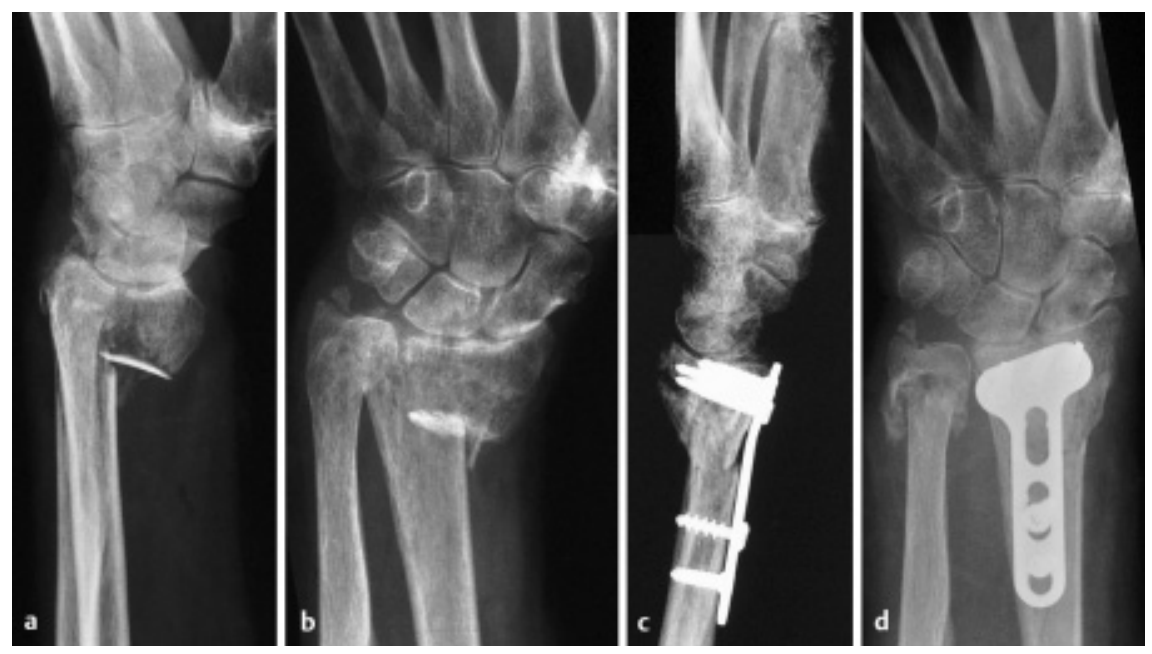

Abb.15a-d Prä- und postoperative Bilder einer A3-Fraktur bei Osteoporose mit palmarer LCP.

$\begin{array}{lr}\text { — exzellent } & 3 \\ \text { gut } & 87 \\ \text { mäßig } & 28 \\ \text { — schlecht } & 0\end{array}$

Erfreulich war die Tatsache, dass auch die Frakturen bei erheblicher Osteoporose, die mit einer LCP versorgt wurden, keine wesentlichen Korrekturverluste im Verlauf zeigten (Abb.15a-e).

\section{Ergebnisse nach $1 \mathrm{Jahr}$}

Zum 1-Jahres-Follow-up konnten bislang Daten von 47 Patienten (Follow-up-Rate 45\%) nach durchschnittlich 369 Tagen (Min 302, Max 446) ausgewertet werden.

Erneut wurden die Patienten zu ihren Schmerzen an der Bruchstelle in Ruhe und bei Bewegung befragt (VAS).
Im Vergleich zu den schon geringen Beschwerden nach 6 Monaten lagen jetzt die Durchschnittswerte der Schmerzen in Ruhe bei 1.2 und bei Bewegung bei 1.7 (Abb.16).

Vergleicht man die Zufriedenheit des Chirurgen und der Patienten mit dem 1.Jahresergebnis, so zeigen die Patienten mit 1.1 eine sehr hohe Zufriedenheit. Die der Chirurgen liegt bei 1.5 (Abb.17).

Im Vergleich zu den Bewegungsausschlägen nach 6 Monaten gab es noch leichte Verbesserungen in allen Bewegungsrichtungen. Gradmäßig bestand die größte Einschränkung bei der Palmarflexion von $13^{\circ}$ zum kontralateralen Arm (Tab.5).

Von bislang 41 auswertbaren Angaben zum Gartland- und Werley-Score konnte folgendes Ergebnis erhoben werden:

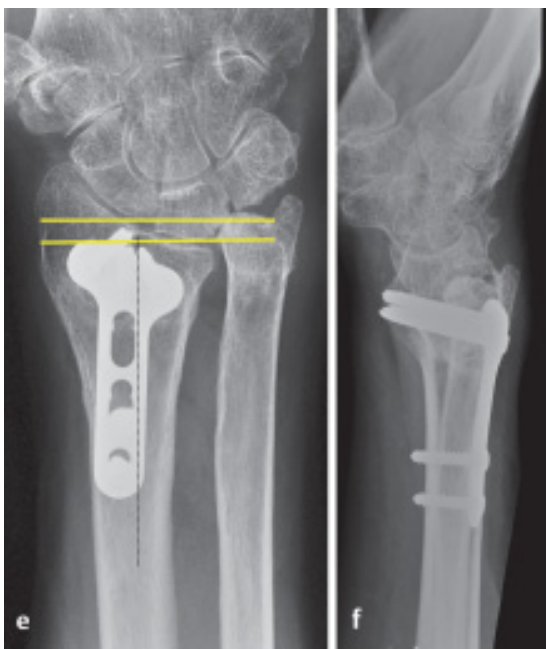

Abb.14e,f Nach 6 Monaten Korrekturverlust mit ulnocarpalem Impingement.

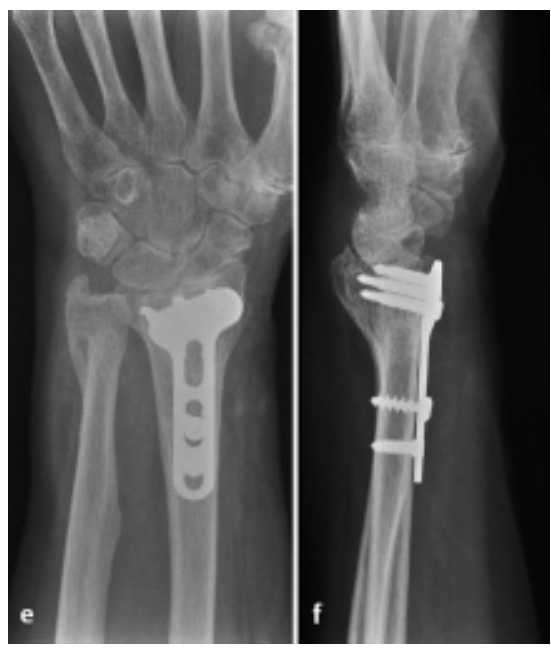

Abb.15e,f Nach 6 Monaten Frakturheilung ohne Korrekturverlust.

$$
\begin{array}{lr}
\text { axzellent } & 0 \\
\text { gut } & 29 \\
\text { mäßig } & 12 \\
\text { schlecht } & 0
\end{array}
$$

Mit dem DASH, „Disability of shoulder, arms and hand"--Fragebogen können Einschränkungen an der oberen Extremität erfasst werden, um Verbesserungen oder Verschlechterungen abzubilden [5].

Ein großer Teil der Patienten erreicht nach einem Jahr gute Werte. Hierbei gilt 0 als der niedrigste bzw. bestmögliche Wert und 100 als der höchste Wert (Abb.18).

\section{Komplikationen}

21 Komplikationen wurden im bisherigen Verlauf bis zum Nachuntersuchungszeitpunkt nach 1 Jahr bei 197 versorgten 


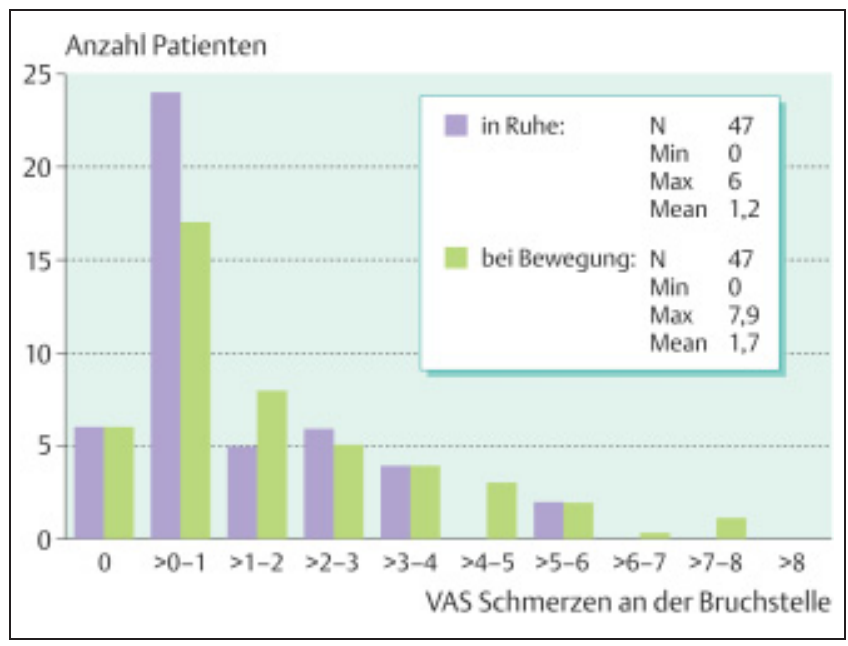

Abb.16 Schmerzen an der Bruchstelle nach 1 Jahr (VAS).

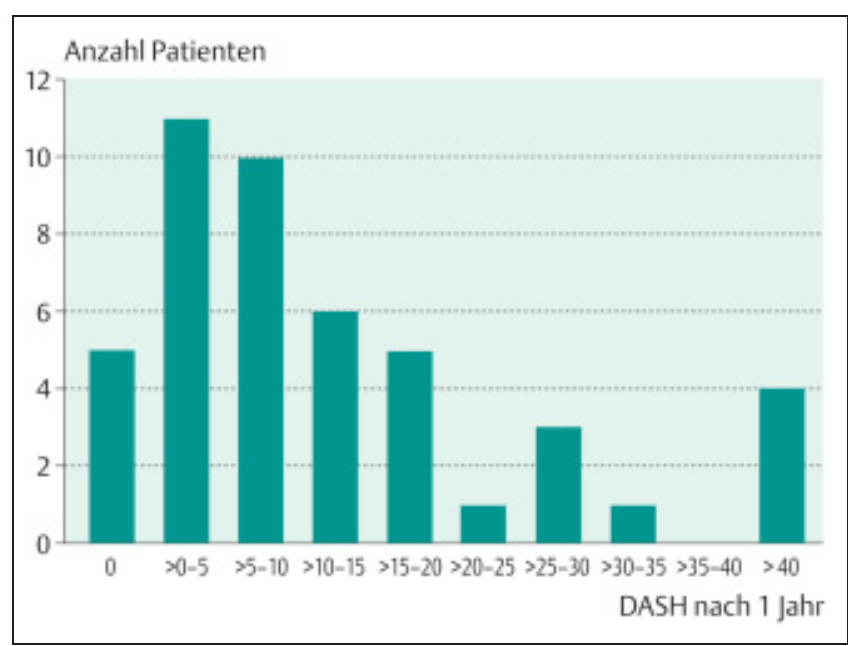

Tab. 5 Bewegungsumfang nach 1 Jahr (R.O.M.)

\begin{tabular}{lcccccc} 
& \multicolumn{3}{c}{ ipsilateraler Arm } & \multicolumn{3}{c}{ kontralateraler Arm } \\
\cline { 2 - 7 } & $\begin{array}{l}\text { Mini- } \\
\text { mum }\end{array}$ & $\begin{array}{l}\text { Maxi- } \\
\text { mum }\end{array}$ & Mean & $\begin{array}{l}\text { Mini- } \\
\text { mum }\end{array}$ & $\begin{array}{l}\text { Maxi- } \\
\text { mum }\end{array}$ & Mean \\
\hline Palmarflexion & 30 & 90 & 54 & 40 & 90 & 67 \\
Dorsalextension & 30 & 85 & 60 & 45 & 90 & 69 \\
Radialduktion & 5 & 45 & 20 & 8 & 55 & 25 \\
Ulnarduktion & 10 & 60 & 32 & 10 & 60 & 36 \\
Pronation & 65 & 90 & 83 & 60 & 90 & 84 \\
Supination & 50 & 90 & 83 & 60 & 90 & 86
\end{tabular}

distalen Radiusfrakturen registriert $(10,8 \%)$. Die operations- und implantatbedingten Komplikationen liegen bei $6,1 \%$.

Im Einzelnen fanden sich folgende Komplikationen:
- 2 Infekte $(1 \%) \quad(1 \times 3,5 \mathrm{~mm} \quad$ LCP. $1 \times 2,7 \mathrm{~mm}$ LCP)

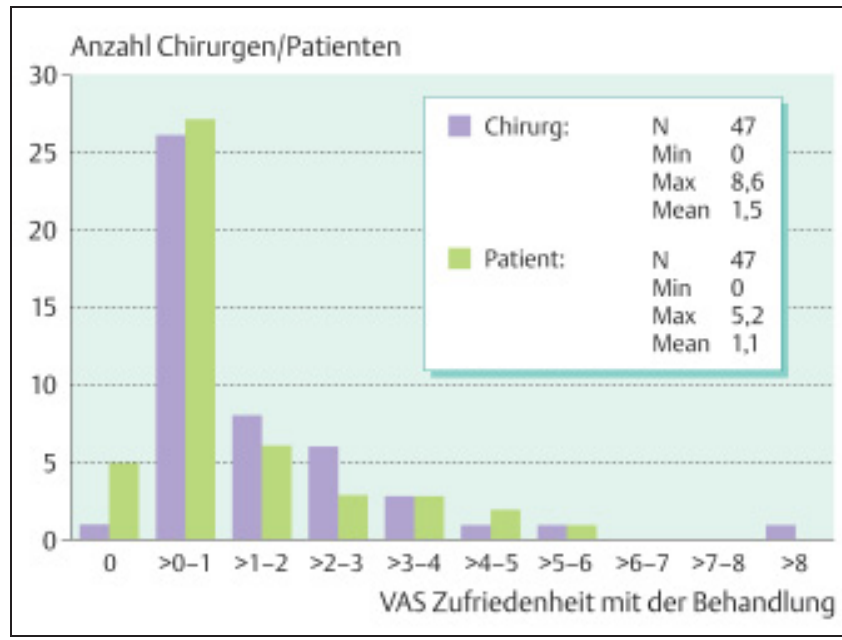

Abb.17 Zufriedenheit Chirurg im Vergleich Patient mit dem Behandlungsergebnis nach 1 Jahr (VAS).
Abb.18 DASH nach 1 Jahr. marer LCP, $1 \times$ dorsale LCP, $1 \times$ PhiPlatte)

2 Strecksehnentendinitis (1\%) PhiPlatte

- 2 CTS $(1 \%)$

- 1 Pseudobursa $(0,5 \%)$

1 Schraubenlockerung (Zugschraube) $(0,5 \%)$

Eine Schraubenlockerung der eingebrachten winkelstabilen Schrauben wurde nicht beobachtet.

\section{Schlussfolgerungen}

Durch die Verwendung der winkelstabilen LCP kam es nur in einzelnen Fällen zu einem postoperativen bzw. sekundären Korrekturverlust.

Eine sekundäre Fragmentdislokation trat in keinem Fall auf.

Trotz einer großen Anzahl von metaphysären Defektzonen (60 A3-Frakturen und 79 C2- bzw. C3-Frakturen) war die Anzahl der durchgeführten Spongiosaplastiken mit $12,2 \%(n=24)$ auffallend gering.

Die Komplikationsrate von Seiten des Implantats mit einer EPL-Ruptur durch eine überstehende Schraube ist sehr klein.

Insbesondere fand sich ein sicherer Schrauben- und Plattenhalt im osteoporotischen Knochen, so dass auch gerade bei diesen Problemfrakturen ein komplikationsloser Verlauf beobachtet wurde.

Als Negativpunkt sind die erhöhten Kosten im Vergleich zur herkömmlichen TPlatte zu sehen. 


\section{Literatur}

1 Jakob M, Rikli D, Regazzoni P. Fractures of distal radius treated by internal fixation and early function. A prospective evaluation. J Bone Joint Surg 2000; 85-B: 340-4

2 Jupiter JB. Current concepts review: Fractures of the distal end of the radius. J Bone Joint Surg 1991; 73-A: 469

3 Lauber P, Pfeiffer KM. Offene Osteosynthese distaler Radiusfrakturen. Resultate und Langzeitverlauf. Unfallheilkunde 1984; 87: 185
${ }^{4}$ Lugger LJ, Pechlander, S. Sehnenrupturen als Komplikation nach Osteosynthese am distalen Radius. Unfallchirurgie 1984; 10: 266 270 ,

5 Mac Dermid JC, Richards RS et al. Responsiveness of the short form-36, disability of the arm, shoulder and hand questionnaire, patient-rated wrist evaluation and physical impairment measurements in evaluating recovery after a distal radius fracture. J Hand Surg (Am) 2000; 25: 330-340

${ }^{6}$ Orbay JL, Badia A, Khouri R, Gonzalez-Hernandez E. Internal fixation for unstable distal radius fractures in the elderly patient. J Hand Surg 2003, Suppl. 1, Vol. 28

7 Wiemer P et al. Frakturen am distalen Radius - Wandel der therapeutischen Strategien. Orthopädie 1999; 28: 846-852
Dr. med. Stefan Matschke Oberarzt

Prof. Dr. med. Andreas Wentzensen Ärztlicher Direktor

BG-Unfallklinik Ludwigshafen Ludwig-Guttmann-Str. 13

D-67071 Ludwigshafen 